\title{
JIM DINE AND THE ARC DETECTOR
}

\author{
Richard Nau \\ Carleton College \\ Northfield MN USA
}

\begin{abstract}
Heart shapes are ubiquitous. Among the numerous places they appear are in the artworks of famous artists as well as children. One of Jim Dine's favorite subjects for print making early in his long career was hearts. Most of his hearts are shaped differently from the usual hearts found in most designs or those given by mathematical formulas. In comparing heart shapes we naturally considered their underlying characteristics, especially those related to smoothness and curvature. To that end, some standard image processing procedures were used, and specialized ones developed and applied.
\end{abstract}

\section{KEYWORDS}

Arc Detection, Heart Shapes, Curvature, Jim Dine

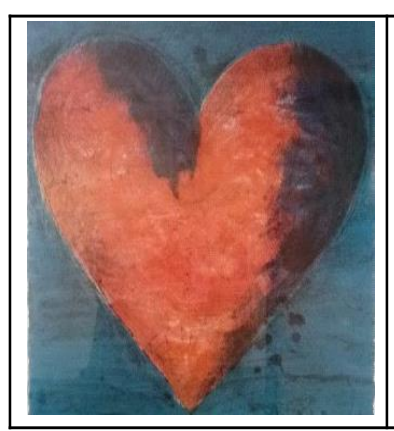

Figure 1. (a) Orange Heart,

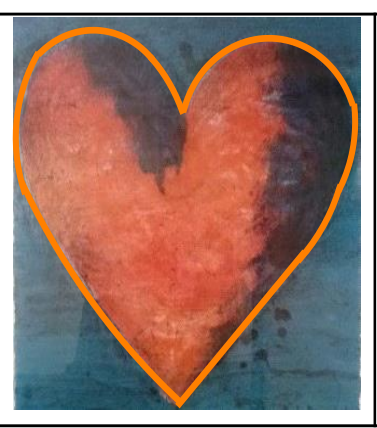

(b) Outline,

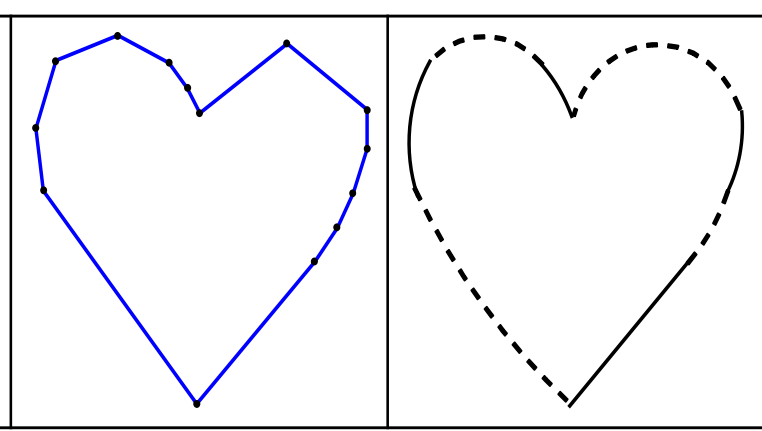

(c) Piecewise Linear,

(d) Piecewise Arcs

\section{INTRODUCTION}

Consider the heart shapes in Figure 1. The one on the left is from a large poster of an orange heart. Its shape is typical of those that appear in many of Jim Dine's 40-some heart prints. His orange heart was selected for its size and because it is relatively well formed as Dine's hearts go. Our goal is to obtain outlines for the shapes, such as shown in Figure 1b, and use them for analytic and comparative purposes. A simple piecewise linear model for the outline is shown in Figure 1c together with the 14 points used. Figure 1d shows a much smoother model using the same points connected by circular arcs. Half of the arcs are dashed for clarification. We will use the term arcs to mean circular arcs, which may simplify to straight line segments, as happened in the longer line at the lower right. It is our contention that the heart shapes in Dine's art can be effectively modeled by such arcs. Of special interest will be the smoothness and curvature exhibited in the models. Although the outline in Figures $1 \mathrm{~b}$ and $1 \mathrm{~d}$ seems satisfactory, one might argue that there were too few points used and that they were not chosen arbitrarily. Using standard techniques of image processing as well as some especially developed routines to detect arcs, we aim to overcome such issues in this paper. First, to establish some bases for comparison, we will consider some heart shapes defined by mathematical formulas. Next we will establish the means for comparing the shapes' features such as smoothness and curvature. Then the routines will be applied to Dine's hearts. Finally, finer points in the methods and artworks will be examined. 
The artworks considered are prints or photographs, and as such there's not a precise location where they are found. D'Oench (see References) incudes 40-some different images for our selection. The two main ones presented here will be referred to as the orange heart and the snowy heart. D'Oench discusses mainly artistic considerations other than shape. Similarly there are many educational articles and projects available that employ Dine's hearts without being particularly concerned with the shape, which in this study is the only concern.

Most of our techniques are familiar in image processing circles. We employed Mathematica for its image processing routines and ability to develop our own. See the References for the author's earlier methods on the radome for the Boeing 747. A recent work (Futterlieb, 2017) studying cloud shapes is similar to ours but on a much larger scale with respect to as the amount of data, the hardware, and the sophistication of its techniques.

\section{DESIDERATA}

Because the interest is in the shape of the curves outlining the hearts, especially considered will be:

1) The fit. How well does the model fit the image? As the piecewise linear model above demonstrates one can fit the points exactly, but still not provide a good outline to the heart.

2) Continuity. Unless there is an obvious discontinuity in the artwork itself, the model should be continuous.

3) Smoothness, i.e. the continuity of the slope of the curve, and hence its tangent and normal vectors. With just a few points as in the example above, it's possible to obtain a model using piecewise arcs that fits the points without the terrible disruptions in the smoothness that the piecewise linear model exhibits.

4) Convexity. The model should be convex wherever the heart shape is. Of course there is the concavity at the cusp at the top that should be met and perhaps some slight concavity near the bottom.

5) Curvature. This will be the foremost objective. Curvature is, by definition, the change in the tangent direction with respect to change in arc length. For a straight line there is no change so the curvature is zero. For a circle the curvature is constant and works out to be the reciprocal of the radius. That's a reason for considering only circular arcs and line segments.

6) Size does not matter. To be able to compare the shapes for hearts of various sizes, we usually will normalize all distances in terms of the radius of the topmost arc of the right lobe, which will be considered to be of radius one. Thus the goodness of fit will be measured by the average absolute deviation between the original points and its approximating curve. Angles and percentages are unaffected by this scaling. Smoothness is measured as the percentage of the turning which is accomplished smoothly.

\section{SMOOTHNESS AND CURVATURE OF SCRIPTED HEART SHAPES}

Figure 2 shows three shapes based on mathematical formulas. Figure 2a shows a heart that commonly is used in elementary schools to familiarize students with circles and lines, perhaps using disks and triangular wooden blocks.. Most all heart shapes include a right lobe and a left lobe and two cusps. The upper cusp we will refer to as the cleft and the lower one as the tip. The exterior angle at the cleft will be denoted by $\alpha$, and the interior angle at the tip by $\beta$. Whereas the elementary heart is upright and balanced those of artists are often slightly askew and asymmetrical. As ones eye traces the right edge of the elementary heart in Figure 2a from top to bottom one reaches the point where the circular arc and the slanted line meet. At this join there's a disturbing discontinuity in the slope, a jerk if you will. The smoothness of that edge is approximately $83 \%$. To determine whether Dine's hearts also often lack smoothness will require close inspection. By matching the tangents at the join the jerk can be removed yielding the "smooth" heart in Figure $2 \mathrm{~b}$. 

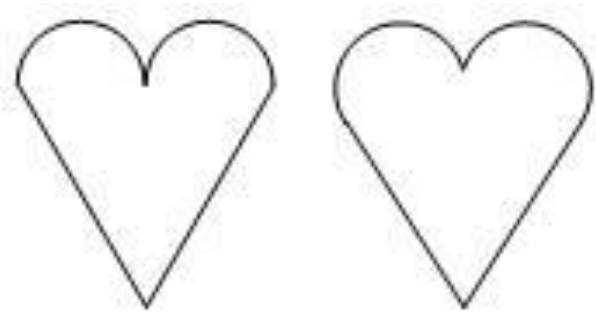

Figure 2. (a) Elementary Heart, (b) Smooth Heart,

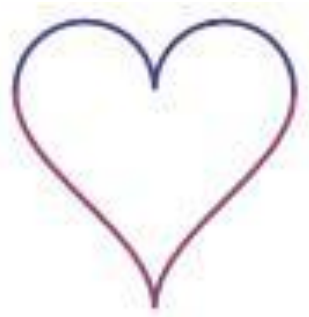

(c) Ideal Heart,

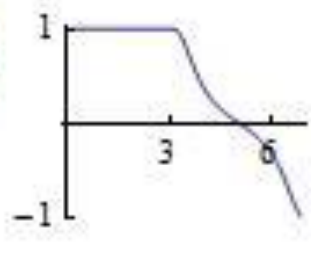

(d) Ideal Heart Profile

The so-called Ideal Heart in Figure 2c combines a semi-circular arc with an arccosine for each lobe. Not only is the curve for each half smooth, i.e. no jerk, but furthermore it has a continuously changing curvature, which among heart aficionados is a highly desired property

Consider again the right lobe of the elementary heart. It starts straight up, bends to the right along the unit circle a distance of $\pi$ units until the joining with the line. From there we travel 4 units straight ahead without curving, i.e. curvature 0 . Thus the motion of the trace for the elementary heart shown can be summarized by $(0,1, \pi),\left(30^{\circ}, 0,4\right)$. A trace in general will be specified as a sequence of triples, $(\Theta, K, L)$, in which $\Theta$ is the clockwise turn, $\mathrm{K}$ (kappa) is the curvature, and $\mathrm{L}$ the distance to be traveled on that segment.

Derivable from the trace is the curvature profile, i.e. the curvature as a function of arc length. This is shown in Figure $2 d$ for the Ideal Heart. The profiles for the elementary and smooth hearts (not shown) are simply step functions. Expressing the curvature in terms of arc length was trivial for the two simpler cases; not so for the Ideal Heart where numerical evaluation of an elliptical integral was required.

\section{MODELING THE DINE HEARTS ${ }^{\circ}$}

Our attempt to model the Dine hearts is intended to remove some of the subjectivity in the choices made. In general, the procedure is to:

1) Obtain an image and crop it to include mainly the heart.,

2) Apply an edge detector.

3) Identify the relevant larger connected components.

4) For each such component, fit a circular arc to it,

5) Assemble the arcs into an outline of the heart.

6) Evaluate the result for fit, smoothness, and curvature.

The sources for the first step varied from a 3 meter square poster of the orange heart to $5 \mathrm{~cm}$ square images in an art book. For steps 2 and 3 we used several of the appropriate routines in Mathematica's Image Processing suite. Steps 4, 5, and 6 will be demonstrated more thoroughly in the context of the next example, which is the snowy heart depicted in Figures $3 \mathrm{a}, \mathrm{b}$ and $\mathrm{c}$. What's being modeled is a snow covered statue. In considering the right lobe notice a short line segment at the cleft, a large circular arc at the top, another short line segment, a large arc on the right side, then a long line towards where the tip would be. The left lobe is less complicated, consisting of a short line, two arcs, and a long line. The edges in the image as detected result in Figure 3b. 


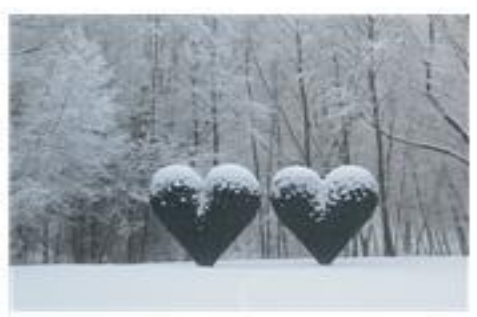

Figure 3. Snowy Heart. (a) Photo,

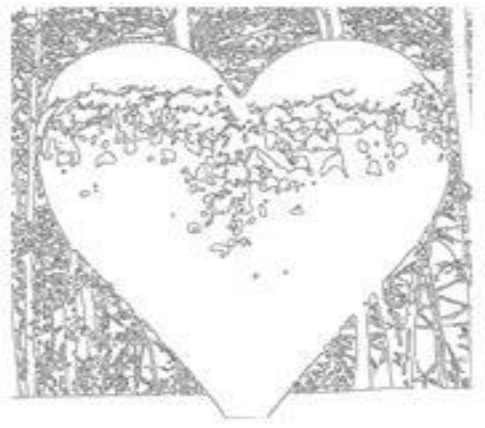

(b) Edges detected,

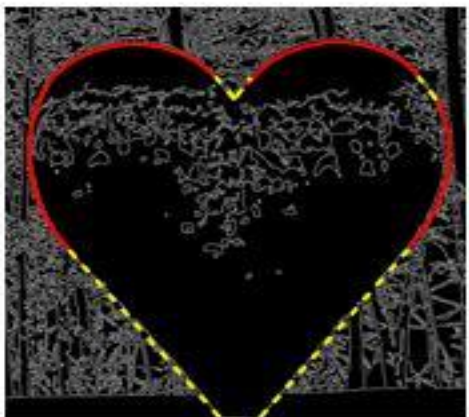

(c) Outline

Then many smaller components were removed. Next the nine major components were identified, dealt with individually and combined into the outline shown in Figure 3c.

The first connected component considered and set was the large arc on the right shoulder. See Figure 4. This component consisted of about 230 points (pixels). It was fit using only five points. If the points selected were too close together, overfitting and scattering could result. So the points used were separated by about 40 pixels. Call these points $1,2,3,4,5$. Three points determine a circle, so the points were grouped $(1,2,3)$, $(2,3,4),(3,4,5)$. Rather than using the equation for a circle one can use the fact that the center of a circle is at the intersection of the perpendicular bisectors of any two chords, as in Figure 5. In this manner three centers could be obtained and averaged to form a common center. The average distance to the five points, or all 230 points for that matter, could be used for the common radius. Although this may seem complicated it is made computationally quite simple by using the generalized matrix inverse.

Suppose there were $\mathrm{n}$ perpendicular bisectors each of the form of a line and assumed to go through the common center $(\mathrm{h}, \mathrm{k})$; i.e, $\mathrm{a} \mathrm{h}+\mathrm{b} \mathrm{k}=\mathrm{c}$. Collectively the set of linear equations as a matrix equation is simply A $(h, k)^{t}=C$, where A is an $n$ by 2 matrix containing columns of a's and b's, C is $n$ by 1 for the c's, and $\mathrm{t}$ denotes the transpose. The generalized solution for this over-determined system is simply $(h, k)^{t}=A^{g} C$ where $A^{g}=\left(A^{t} A\right)^{-1} A$ is the generalized matrix inverse. Please note this works very efficiently for any $\mathrm{n}(>=2)$.

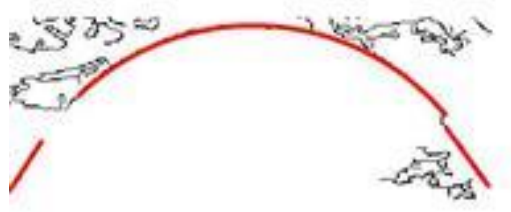

Figure 4. Snowy right top.

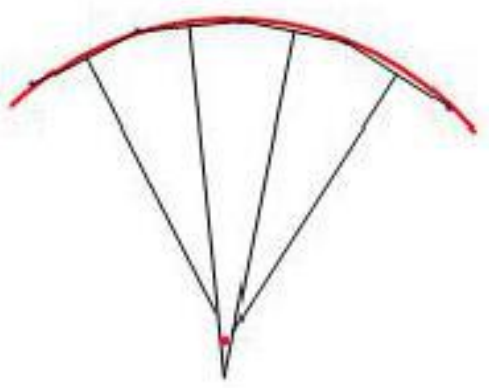

Figure 5. Four bisectors.

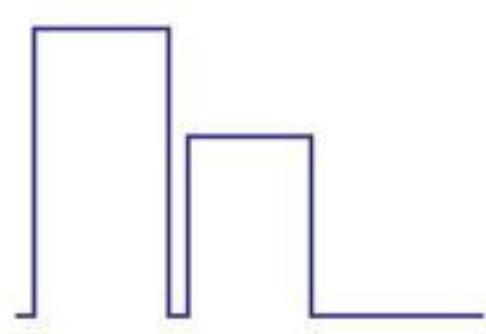

Figure 6. Snowy Right Profile

The bulb at the left lobe was handled similarly to that on the right. The connected components for the two long lines at the tip can be easily dispatched. Shorter ones require more explanation. Consider the two short components in the lower corners of Figure 4. If the arc were extended it would undershoot the one on the left and overshoot the one on the right. These situations are often referred to as an under-bite and an overbite, resp. The short component at the left in Figure 4, i.e. the one near the cleft, when blown up reveals its linear fit. Any closer fit would result in a concavity, which is unjustified considering the few points involved. The connections between the top arcs and the long lines required some intervention. Looking closely at those areas it is clear that those components are not as well defined as for the top arcs and the long lines. A single 
arc on the left lobe connected its top arc and long line. On the right lobe an intervening short line segment was discerned there. The curvature profile for the right lobe showing the curvature is given in Figure 6.

In analyzing other Dine hearts there were many similarities. The larger hearts were generally elongated and had a right lobe slightly larger than the left and were tilted by a few degrees to the right which gave an illusion that added to the apparent asymmetry. Again large components were obtained and fitted by arcs with little error. Joining the arcs often required compromises among the fit, curvature and smoothness. However in all cases the fitness on the main components was within a percentage or two and the amount of jerkiness limited to less than $20 \%$, Smaller hearts studied were often so well formed that the largest component was in fact an entire outline by itself and the main task was to separate it into adjacent circular arcs.

\section{RESULTS AND CONCLUSIONS}

Applied to Dine's hearts the generalized matrix inverse based arc detector proved to be a very efficient and effective tool for obtaining the arcs and their curvature as well as to measure their fitness. It would be informative to apply it to more artworks and other areas where circular arcs are known to exist or suspected.

Visually piecewise arcs, requiring very few arcs, fit the heart images tested, at least their major edge detected components. It's not a surprise that arcs would fit, but what is surprising is how few were sufficient, how few points were needed from each arc, and how well they fit, often within $1 \%$. .Furthermore, the resulting curvatures were constant over fairly large regions. Trade-offs between the fit and smoothness were possible although a smoothness of at least $80 \%$ was always achievable.

More accuracy and smoothness could be obtained using more arcs. One could also eliminate some of the jerkiness by sacrificing some fit. However closer examination shows that much of that jerkiness is really there, i.e. it is an artifact in the art, not a consequence of the algorithms. We should not blame the algorithm. Even more fit could be expended to get a more smoothly changing curvature as well. But generally, the tight fit indicates that that curvature obtained, or something very close to it, is really there.

Some smaller anomalies in the artworks were detected, such as a slight discontinuity or a small unexpected concavity. Although the larger arcs and longer lines were well defined, where they joined was often somewhat obscure. Indeed a detailed inspection of the artworks reveals that those areas were subjected to shadowing, over-sketching and what might be considered patching. But that, together with such irregularities as the tilting and asymmetry, is what makes it art rather than computer graphics. Jim Dine, when asked about the rationality behind his work is said to have quipped "I have never had a rational thought".

\section{ACKNOWLEDGEMENT}

The support of Carleton College to present this work is greatly appreciated. I also wish to thank the reviewers for their thoughtful and constructive advice.

\section{REFERENCES}

D’Oench, E.G. and J. E. Feinberg, 1986. Jim Dine Prints 1977-1985, Harper \& Row, New York, NY, USA.

Futterlieb, J. et al, 2017. Smooth Visualization of Large Point Clouds. IADIS Journal on Computer Science and Data Visualization Vol 11, No 2, Porto, Portugal, pp 146-156.

Nau, R, 1966. Generalized Radome Incidence Analysis. Boeing Reports, Seattle, WA, USA. 